\title{
Traduire
}

Revue française de la traduction

L'environnement, une spécialisation durable?

\section{Les clichés en traduction : les jurons irlandais}

\section{Pierre Fuentes}

\section{(2) OpenEdition}

Journals

Édition électronique

URL : http://journals.openedition.org/traduire/592

DOI : 10.4000/traduire.592

ISSN : 2272-9992

\section{Éditeur}

Société française des traducteurs

Édition imprimée

Date de publication : 15 décembre 2013

Pagination : 67-80

ISSN : 0395-773X

\section{Référence électronique}

Pierre Fuentes, «Les clichés en traduction : les jurons irlandais », Traduire [En ligne], 229 | 2013, mis en ligne le 15 décembre 2015, consulté le 01 mai 2019. URL : http://journals.openedition.org/traduire/592 ; DOI : 10.4000/traduire.592 


\section{Les clichés en traduction : les jurons irlandais}

\section{Pierre Fuentes}

Les clichés de l'Irlande ont de beaux jours devant eux. En témoigne le succès de films qui exploitent le filon du stéréotype, comme le récent L'Irlandais (The Guard, 2011). Jouant essentiellement sur le choc des cultures, le scénario met en scène la rencontre d'un flic irlandais, blanc, très attaché à son comté de Galway et particulièrement relax sur les procédures, et d'un agent fédéral américain, noir, d'un milieu social privilégié et très à cheval sur les principes. L'humour de cette " comédie corrosive qui ne polit pas les angles du politiquement correct " (selon les mots de Christophe Carrière dans L'Express), ne cherche pas à surprendre le public non irlandais mais, au contraire, répond à ses attentes. Une scène présente par exemple un irlandophone qui refuse ostensiblement de s'exprimer en anglais lorsque l'agent du FBI lui demande un renseignement. Si cette attitude a dû ravir le spectateur soucieux de défendre la richesse d'un pluralisme culturel et linguistique face au spectre de l'impérialisme anglo-saxon, il s'agit néanmoins d'un stéréotype attendu par une audience qui n'est pas familière du comportement sociolinguistique des Irlandophones. Certes, des communautés irlandophones subsistent dans le Connemara, mais leurs locuteurs ne s'adressent jamais aux étrangers en irlandais (surtout aux Américains qui sont traditionnellement perçus comme une source de revenus touristiques). Comme le résume la sociolinguiste Bernadette 0'Rourke (2011), le comportement des irlandophones natifs des Gaeltachtaí(1) est le reflet d'une norme sociale identifiée par plusieurs enquêtes à grande échelle : ils parlent anglais avec les étrangers. Cette posture peut être perçue comme le résultat d'une attitude " très ouverte "à l'égard de ceux qui sont incapables de parler irlandais - ou qui s'y refusent - mais elle témoigne avant tout d'une forme de résignation car les irlandophones ne perçoivent pas leur langue comme un signe de supériorité sociale (ibid. p. 342).

Il existe pourtant de nombreux auteurs irlandais qui refusent le cliché - ou qui se servent du cliché pour railler ceux qui en abusent. Leur œuvre constitue un véritable défi pour le traduc-

(1) Les irlandophones parlent le gaélique irlandais (gaeilge). Les autres langues gaéliques comprennent l'écossais (gàidhlig) et le mannois (gaelg). Le terme "Gaeltacht " désigne les zones de l'Irlande où l'irlandais est la langue principale de la population locale. Les Gaeltachtaí bénéficient d'un statut officiel. 
teur. Seul face à l'océan sémantique du texte source, il se précipite souvent sur cette bouée de sauvetage, seul point d'appui visible qui lui permette de soulever le poids du sens. En effet, le cliché est le premier point de contact entre culture source et culture cible. Le traducteur doitil l'éviter ? Peut-il l'éviter? Afin d'aborder ces questions, le présent article tente d'analyser un aspect spécifique de la traduction française d'un roman irlandais : la traduction des jurons profanes en tant que stéréotype de la très catholique Irlande(2).

\section{La mécanique du cliché}

Les clichés peuplent notre mémoire collective. À l'époque de la photographie argentique, le cliché désignait l'épreuve du support négatif. À partir d'un seul cliché photographique, on pouvait obtenir un grand nombre d'impressions sur support papier. Avec l'avènement du numérique, ce chiffre est devenu virtuellement infini. II en va de même pour le cliché verbal. Au sens figuré, le cliché est une expression verbale toute faite devenue stéréotypée à force d'être répétée. Qu'il soit réel ou figuré, le cliché constitue un véhicule de l'idée, voire de l'idéologie concept que le journalisme confond parfois avec l'information. Les innovations du numérique ont donc permis de démultiplier l'idée à l'infini et presque immédiatement, qu'elle soit verbale ou visuelle. Comme le proclamait Paul Virilio (1996, p. 12), avec l'avènement de l'ère numérique, "l'histoire vient d'emboutir le mur du temps ". Chaque nouveau cliché contamine notre mémoire collective désormais instantanément, que nous soyons journaliste, traducteur ou simple internaute.

Le cliché est une forme de stéréotype, au sens où l'a défini Dufays (2001, p. 25), car il affecte notre perception de la réalité à trois niveaux : linguistique (l'expression cliché), formel (le cliché du genre) et idéologique ('a priori). Dufays énumère ainsi plusieurs caractéristiques du stéréotype : 1 . le stéréotype se rigidifie : ses composants font l'objet d'un semi-figement, entre eux et envers le contenu qu'ils désignent ; 2 . il se caractérise par une fréquence d'emploi, une pérennité et un caractère collectif ; 3 . il est anonyme et 4 . le stéréotype est employé par automatisme (ibid. p. 26). Pour Dufays, ces conditions ne sont pas suffisantes pour qu'un phénomène relève de la stéréotypie. II faut encore que le phénomène en question fasse problème du point de vue référentiel (cliché simpliste, réducteur ou erroné), psychologique (manque de discernement), informationnel (banalité dénuée de valeur) ou éthique (cliché injuste, voire discriminatoire). Comme il le souligne, ces problèmes ne sont évalués qu'au moyen de notre jugement personnel, qui est par nature subjectif. Par conséquent, dès lors que nous défendons une idée, nous nous exposons au reproche de stéréotypie. La chanson et le cinéma ne nous ont-ils pas avertis qu'" on est toujours le con de quelqu'un(3) " ?

(2) Le 20 février 2013, une recherche en ligne de "la très catholique Irlande " obtenait, avec les guillemets, 21400 résultats.

(3) Cf. entre autres, les chansons de George Brassens et de Pierre Perret ou Le dîner de cons de Francis Veber. 


\section{The Snapper}

Publié en 1990, The Snapper est le second volet de la trilogie de Barrytown de l'auteur dublinois Roddy Doyle. L'auteur tire un effet humoristique, mais non caricatural, de la variété d'anglais parlée par ses personnages. Ses romans traitent de sujets sérieux, voire graves, mais avec légèreté et en respectant la dignité des couches sociales qu'il met en scène; ils appartiennent au réalisme social, non à la satire. Constitués en grande partie de dialogues, ils se prêtent facilement à l'adaptation audiovisuelle ou au théâtre. Le troisième volet de la trilogie, Les Commitments a été adapté au cinéma en 1991 par Alan Parker(4). II a rencontré un grand succès dans les salles, en partie grâce à sa bande originale. En revanche, l'adaptation cinématographique de The Snapper, par Stephen Frears en 1993, n'a bénéficié que d'une faible médiatisation. À l'époque, le film est tout de même sorti dans les salles françaises, en version sous-titrée. L'une des caractéristiques des dialogues de la trilogie de Barrytown est l'utilisation répétée de jurons " religieux " qui ont probablement constitué une difficulté pour le traducteur français, Bernard Cohen.

The Snapper raconte l'histoire de Sharon Rabbitte, une Dublinoise âgée de vingt ans, qui se retrouve enceinte. Bien qu'elle ne veuille pas leur révéler qui est le père de l'enfant, son père et sa famille sont déterminés à la soutenir. La traduction française a été publiée en 1996, puis à nouveau en 2009, chez Laffont. Elle demeure, semble t-il, la seule traduction française disponible à ce jour. Suite au succès des Commitments et au prix Booker attribué à Roddy Doyle en 1993 pour Paddy Clarke Ha ha ha, la critique internationale a fini par s'intéresser à The Snapper. Toutefois, au moment de la première édition, le roman s'adressait principalement à un public irlandais qui avait un accès direct au contexte socioculturel. Depuis, ce contexte a énormément évolué : après avoir traversé une période de boom économique sans précédent, la république a été particulièrement touchée par les affres de la crise financière de 2007. Comme la Grèce ou le Portugal, sa dette souveraine a fait l'objet d'un renflouement par la BCE et le FMI. L'austérité économique actuelle touche de nombreuses couches de la société et, aux classes ouvrières traditionnelles, dont Roddy Doyle brossait le portrait, sont venus s'ajouter de nouveaux pauvres. Sur ce fond de désespoir économique a éclaté une autre crise : le 20 mai 2009, la Commission Ryan a publié un rapport(5) sur des cas de violences psychologiques, physiques et sexuelles faites à l'encontre de milliers d'enfants dans le milieu scolaire catholique entre les années 1930 et 1990 (précisons qu'en Irlande, la plupart des écoles, publiques ou non, étaient gérées en majorité par le clergé catholique). Si la religion catholique avait déjà

(4) Et Doyle vient de l'adapter une énième fois dans le cadre d'une comédie musicale pour le West End londonien.

(5) Commission report, Commission to Inquire into Child Abuse, 20 mai 2009 :

http://www.childabusecommission.com/rpt/ExecSummary.php 
perdu son autorité au sein des classes ouvrières traditionnelles dans le Dublin de Doyle, ce n'était peut-être pas un fait visible pour le public français de 1996. II faut espérer que depuis 2009, ce n'est plus le cas.

\section{Destruction du vernaculaire et dérive}

Depuis le célèbre essai de Friedrich Schleiermacher (1813/1963), les traducteurs savent qu'ils sont confrontés à la tâche difficile de transmettre au lecteur, d'une façon ou d'une autre, l'étrangeté qu'ils ont eux-mêmes rencontrée au cours de leur lecture d'un texte original. Le philosophe allemand évoquait une posture qui vise à laisser à nu le gros-œuvre de l'original ayant servi à " cintrer " la traduction (ibid. p. 55). Deux siècles plus tard, le concept avait muté sous la plume de Lawrence Venuti (1995) : à l'approche traditionnelle qui consiste à domestiquer le texte étranger, il opposait sa fameuse foreignization (altérité). En France, la domestication restait l'approche dominante depuis l'époque de Joachim Du Bellay (Berman, 1986). Quelles qu'aient été les tentatives de comprendre, ou de prescrire, la posture du traducteur à travers le monde et les époques, elles ont été invariablement motivées par une idéologie sous-jacente : Eugene Nida, par exemple, était pasteur et travaillait à répandre la parole divine ; Lawrence Venuti dénonce pour sa part l'invisibilité que le marché américain de l'édition impose aux traducteurs.

Pourtant, " la seule question quand on écrit, c'est de savoir avec quelle autre machine la machine littéraire peut être branchée, et doit être branchée pour fonctionner " car " la littérature est un agencement, elle n'a rien à voir avec de l'idéologie, il n'y a pas et il n'y a jamais eu d'idéologie ", selon la célèbre formule de Deleuze et Guattari (1980, p. 10). Pour Antoine Berman (1985/2004, p. 278-279), cet agencement, lorsqu'il subit l'épreuve de l'étranger, fait l'objet d'une " atténuation ", car les traducteurs sont toujours victimes d'un désir de traduire motivé par leur inconscient. Or la psychanalyse nous l'a appris, nul ne peut totalement censurer son inconscient, qu'il soit d'origine collective ou individuelle. Parmi ces pulsions inconscientes, l'une des atténuations identifiées par Berman est la " destruction du vernaculaire " (ibid. p. 280). The Snapper contient une forme de vernaculaire car il met en scène des personnages qui s'expriment dans une variété d'anglais, parfois désignée sous le nom d'hiberno-english (anglais irlandais). II est donc probable que la traduction de Cohen n'ait pas pu échapper à une certaine destruction du vernaculaire qu'emploie Roddy Doyle.

Venuti (1995, p. 17-29) décrit lui aussi les choix effectués par les traducteurs comme relevant partiellement d'un processus inconscient. S'éloignant de la tradition humaniste, il cherche à démasquer, par le biais d'une lecture symptomatique (symptomatic reading), les tendances de domestications présentes dans les traductions qui se revendiquent pourtant de l'altérité, en montrant que le caractère d'étrangeté dépend avant tout de points d'appuis culturels domestiques. Mais contrairement à Berman, il conçoit l'acte de traduction comme un processus contrôlable qui doit perturber la fluidité de la lecture (1995, p. 307-313) en s'appuyant sur la 
" dérive ", terme qu'il emprunte au romancier Maurice Blanchot (1971, p. 71). Cette perturbation peut, et doit être effectuée, affirme-t-il, au moyen d'archaïsmes, de l'argot, d'allusions et de conventions littéraires qui attirent l'attention du lecteur sur le statut secondaire de la traduction et identifient les divergences culturelles par rapport au texte original (Venuti, 1995, p. 310-311).

Notre analyse cherche donc à démasquer le caractère domestique des stratégies utilisées par Cohen pour traduire un élément spécifique du roman de Roddy Doyle : les jurons à caractère religieux.

\section{Jaysis ! Jesus ! And God !}

Dans son Petit livre des gros mots, Gilles Guilleron (2007) compare les expressions " maison de tolérance, matière fécale ! " et " bordel de merde ! " : pour lui, les deux expressions transmettent une signification en apparence identique, mais la seconde est porteuse d'une charge émotive car elle appartient au registre des grossièretés. Toutefois, " on peut être extrêmement grossier sans dire un seul gros mot(6) " : définir la grossièreté est un exercice périlleux qui ne touche pas seulement à la linguistique mais aussi à la sémantique. La linguiste Ruth Wajnryb (2005a) s'y était risquée et soulignait qu'il existait deux pièges dans l'étude du langage grossier : le premier tient aux mots mêmes qui constituent la grossièreté, un même mot pouvant être utilisé dans des contextes différents et produire des sens différents, et le second à ce que nous percevons comme grossier, le sens pouvant être porteur de valeurs différentes (malédiction, insulte, obscénité, blasphème, etc.). Ainsi, les jurons ne se prêtent pas aisément à la traduction (Wajnryb 2005b, p. 177). II est donc très difficile pour les traducteurs de choisir entre les diverses stratégies qui s'offrent à eux car aucune d'entre elles ne sera totalement réussie.

Notre analyse porte sur les termes Jesus, Jaysis, et God lorsqu'ils ont été utilisés en tant que grossièreté profane, une forme de jurons que Wajnryb (2005, p. 15) distingue du blasphème par l'usage qui est fait du terme sacré. Le blasphème consiste à moquer la divinité, et à travers elle, la religion, ce qui, pour les Juifs, les Chrétiens et les Musulmans, va à l'encontre du droit religieux et en particulier du troisième commandement : " Tu ne prononceras pas à tort le nom du Seigneur, ton dieu, car le Seigneur n'acquitte pas celui qui prononce son nom à tort(7) ". Au contraire, les grossièretés profanes n'ont pas pour intention de moquer la religion, que la personne qui les professe soit croyante ou non. Les jurons religieux peuvent aussi être proférés à des fins amicales, comme le souligne David Crystal (1995, p. 173), lorsqu'une

(6) Un air de famille, Agnès Jaoui et Jean-Pierre Bacri, 1994.

(7) Exode, 20, 7, Traduction œcuménique de la Bible, Société biblique française et Éditions du Cerf, 1988, p. 99. 
personne s'identifie à un groupe et qu'elle veut s'y intégrer. Dans un tel cas, ces grossièretés ne contiennent pas de connotation négative, ou presque, et s'apparentent même à une forme de convivialité.

Le degré de vulgarité d'une grossièreté, en revanche, varie suivant la perception de chacun. En anglais britannique, par exemple, Jesus est considéré comme relativement vulgaire et reste plus employé par les hommes (McEnery 2006). À l'opposé, God est perçu comme familier et plus utilisé par les femmes. L'une des conclusions de cette étude est que l'usage utilise les grossièretés comme une marque de distinction sexuée, les hommes britanniques faisant généralement appel à un vocabulaire perçu comme plus grossier que celui des femmes (ibid. p. 246). Néanmoins, l'étude de McEnery porte sur un usage purement britannique. II est possible que des résultats différents soient obtenus avec un échantillon d'anglophones irlandais. En effet, une étude basée sur le Limerick Corpus of Irish-English (Farr 2008), a montré que le fait de jurer était profondément enraciné dans la culture irlandaise. Ce comportement pourrait être la conséquence de la manière dont l'anglais a été imposé à cette population : les colonisés ont d'abord appris le langage que les colons utilisaient avec eux.

Quoi qu'il en soit, dans les deux variétés de langues, les termes Jesus et God désignent dans leur sens premier le dieu de la Bible. Ce n'est que lorsqu'ils sont employés comme interjections, que leur sens migre du sacré vers le profane. II se produit alors une " dé-sémantisation du sens " (Siblot 1995) : employés comme interjections, ces jurons sont vidés de leur sens premier ; leur fonction expressive "se borne à marquer une intensité émotive " (ibid. p. 166). Dans la bouche des personnages du Snapper, ils perdent d'ailleurs toute connotation sacrée. S'il fallait s'en convaincre, il suffirait de poser la question à l'auteur, qui affirmait que la religion n'a pratiquement pas droit de cité parmi les classes ouvrières dublinoises :

I wanted to get away from the cliched view of Ireland. An English critic of The Snapper said: "Where was the priest? This is a pregnant girl." And I wanted to say, "Fuck you pal - what do you know ? You live in London." Priests in working-class parts of Dublin are peripheral figures few people know who they are at all, and they're not particularly welcome when they knock on the door(8).

Quant à l'orthographe Jaysis, Roddy Doyle l'utilise pour souligner la prononciation dublinoise de Jesus, quoi qu'il n'en soit probablement pas l'auteur. Elle peut certainement être considérée comme irlandaise : à titre indicatif, une recherche du terme sur le moteur de Google au 22 février 2013 obtenait 150000 résultats sur les pages irlandaises uniquement, contre 45000 pour les pages britanniques uniquement, soit un usage trois fois supérieur pour une population nationale vingt fois moins nombreuse.

(8) Roddy Doyle, cité dans Reading Roddy Doyle, Caramine White, 2001, p. 169 : " Je voulais échapper au cliché habituel de l'Irlande. Un critique anglais avait dit à propos du Snapper: "Où est le prêtre ? II s'agit d'une fillemère". J'avais envie de lui répondre "Va te faire foutre, mon pote ! Qu'est-ce que t'y connais ? Tu vis à Londres". Dans les quartiers ouvriers de Dublin, les prêtres sont tout au plus des figurants ; peu de gens les connaissent et ils ne sont pas particulièrement bienvenus quand ils frappent à la porte. " [Ma traduction]. 
La question qui nous occupe est donc de savoir quelles ont été les stratégies de Cohen, visà-vis de la traduction de ces trois interjections et si ses traductions échappent au cliché auquel Roddy Doyle voulait lui-même échapper.

\section{Des jurons en français}

Ce sont 190 termes ayant trait à la religion qui ont été relevés dans le texte original. Bien que la proportion s'élève à presque une référence par page, leur distribution n'est pas homogène. Elle dépend de la teneur et du registre de langue utilisés par les personnages mis en scène, et se concentre donc dans certains passages. Parmi ces 190 occurrences, les termes Jesus, Jaysis, et God sont très majoritaires. Considérées dans leur contexte original, un petit nombre d'entre elles se produisent à l'intérieur d'expressions idiomatiques telles que every jaysis one o' them (Doyle, 1990, p. 144). Ces expressions n'étant pas employées en tant que jurons, nous les avons laissées de côté. En revanche, la plupart des occurrences restantes constituent des jurons profanes, au sens où nous l'avons défini. Les tableaux 1, 2 et 3 donnent une liste des dix premières stratégies employées par Cohen pour traduire Jaysis, Jesus et God, y compris avec leurs variations. Les stratégies les plus récurrentes sont "De Dieu ! ", "Doux Jésus ! ", et "Mon Dieu! ", mais il faut souligner que les variations sont nombreuses et semblent liées aux variations du juron présent dans le texte original, par exemple Sweet Jesus !, Good Jaysis ! et my God!

Premier fait saillant, ces tableaux montrent que Cohen s'est efforcé de traduire les jurons religieux de Doyle par des jurons en français. Soulignons-le, il s'agit de "jurons en français ", et non de " jurons français ", car il s'agit bien de mots appartenant au dictionnaire français mais le fait que leur identité française soit reconnue par le lecteur de France reste à prouver. En effet, la manière dont nous jurons est déterminée par notre culture (Wajnryb, 2005b, p. 136) et les jurons traditionnels d'une variété particulière de français ne sont pas nécessairement les mêmes que ceux d'une autre variété. Ainsi, les attentes d'un public francophone de France sont différentes de celles d'un public francophone d'une autre culture. S'il est possible qu'il connaisse des jurons tels que " tabarnak! " ou " fât' pêtard! ", il est fort probable que le public français s'attende à des jurons plus usités en France, tels que ceux ayant trait à la prostitution (" putain ! " ou " bordel! ") ou à la scatologie (" merde ! " ou " 'fait chier ! "). Or, la traduction de Cohen s'adresse avant tout à un lecteur dont l'usage du français s'apparente en priorité à celui de France. L'éditeur de la traduction française du Snapper, les éditions Robert Laffont, visait avant tout son marché traditionnel, la France, ce que confirme le choix de son traducteur : Bernard Cohen est en effet journaliste et traducteur français.

\section{Archaïsmes}

Seconde observation qui ressort de ces données : Cohen s'est employé à utiliser des jurons religieux. Dans la majorité des cas, sa stratégie consiste à employer les termes "Dieu ", 
"Seigneur " et " Jésus ", des traductions dont l'archaïsme pourrait indiquer une possible intention d'altérité. Néanmoins, qualifier ces jurons d'archaïsmes constitue en soi un jugement subjectif, que l'on pourra taxer de stéréotypie (Dufays 2004). II convient d'abord de les examiner dans leur contexte, puis de les examiner au regard de l'évolution de l'usage linguistique du français. À cette fin, voici un exemple, tiré du premier chapitre :

Sharon was pregnant and she'd just told her father that she thought she was [...]

- Oh - my Jaysis, said Jimmy Sr.

(The Snapper, Roddy Doyle, 1990, p. 1)

Sharon était enceinte et venait juste d'annoncer à son père qu'elle pensait l'être [... ]

- Oh... Doux Jésus ! Articula Jimmy Sr.

(The Snapper, Roddy Doyle, traduit par Cohen, 2009, p. 9)

Nous l'avons souligné, les jurons religieux profanes n'ont pas de valeur blasphématoire. Ce n'était cependant pas le cas à l'origine, c'est-à-dire à l'époque où ils sont apparus dans le langage. En effet, l'évolution de leur usage est intimement liée à l'évolution du statut de la religion et de son clergé. Si le but premier de ces jurons était d'enfreindre la loi de Dieu, ces jurons ont continué d'être utilisés, dans les pays où l'Église est restée tardivement partie prenante des affaires de l'État, comme en Irlande, au Québec ou au Royaume-Uni, parce qu'ils avaient valeur d'exutoire : il ne s'agissait plus de blasphémer (enfreindre la loi de Dieu), mais de pester (manifester son mécontentement par des paroles violentes) entres autres contre un clergé qui se mêlait de toutes les sphères de la vie sociale. Néanmoins, si les Québécois d'aujourd'hui utilisent des "sacres " en place des jurons de France, cela ne les rend par pour autant plus croyants que les Français. Les études le prouvent : certes $83 \%$ de Québécois sont catholiques (Statistiques Canada 2011) contre $64 \%$ de Français (IFOP. 2007), mais seuls $10 \%$ des catholiques québécois se disent pratiquants (Vallée 2007). Comme dans le cas irlandais, affirmer que les Québécois utilisent des jurons à caractère religieux parce qu'ils sont catholiques relève du cliché éculé. L'usage des jurons religieux au Québec n'est pas le fait d'une population très croyante mais un effet de l'évolution de l'usage qui peut être perçu comme une spécificité culturelle.

En France, la séparation de l'Église et de l'État ayant été consommée depuis longtemps, cet exutoire était moins nécessaire et les jurons religieux ont migré beaucoup plus tôt vers un usage profane (interjections). Or, le propre de l'usage est d'user. L'usure touche tous les registres d'une langue, à plus ou moins longue échéance. Les jurons sont les premières victimes de cette érosion car, nous l'avons vu, ils sont porteurs d'une charge émotive. Ils se dévalorisent donc progressivement, leur emploi en tant qu'interjection marquant l'avant dernière étape de la dé-sémantisation (Siblot 1995). À ce stade, ils ne peuvent subir qu'un ultime affront avant de disparaître, celui de perdre leur statut de jurons. Ainsi, pour Littré (1873), les jurons faisant intervenir "Dieu ", " Jésus " ou "Seigneur " n'étaient déjà plus que des " interjections ", voire des " exclamations ". Le célèbre lexicographe français, pour qui "merde ! " était une " inter- 
jection basse et grossière " et pour qui " putain! " n'existait pas encore en tant que juron, n'attribuait aucune vulgarité, grossièreté, bassesse, aucun caractère populaire, ni même familier au registre des jurons religieux. Plus proche de nous, Le Grand Robert (2001) n'accorde pas non plus le statut de juron à l'interjection "Doux Jésus ! ", et va jusqu'à la qualifier de " courante naguère, surtout en milieu rural".

Si nous hésitons encore à qualifier " Doux Jésus ! " d'interjection française archaïque, nous pouvons à tout le moins admettre qu'elle n'est pas naturelle, au xxe siècle, dans la bouche de cet ouvrier du bâtiment qu'est le père de Sharon Rabbitte. Cet emploi ne peut que perturber la fluidité de la lecture, ce qui, comme nous l'avons souligné, est le propre de la stratégie d'altérité (Venuti 1995).

Toutefois, les guides touristiques et la culture hollywoodienne, que nous pouvons considérer comme des points de contact interculturels sinon universels du moins dominants, ne cessent d'associer l'Irlande au catholicisme, et renforcent les clichés qui hantent notre perception de ce pays. Nous avons aussi évoqué le fait que la religion est un cliché que Doyle voulait éviter dans son œuvre (White 2001, p. 169). Nous pouvons donc arguer que faire appel à des jurons archaïques religieux pour traduire les jurons profanes originaux renforce le lecteur dans sa perception du cliché. En d'autres termes, cette approche d'altérité s'appuie avant tout sur des références culturelles domestiques.

Enfin, il convient de noter que dans le cas de Jaysis, aucune stratégie particulière n'a été adoptée pour distinguer sa traduction de celles de Jesus, ou de God. Comme le montrent les données des tableaux 1, 2 et 3 , les traductions respectives de ces trois jurons se mêlent indistinctement dans le texte de Cohen. Ces résultats prouvent que le traducteur n'a pas cherché à distinguer le terme vernaculaire du terme anglais habituel. Ils constituent, selon les mots de Berman (1985/2004, p. 286), un effacement, ou une destruction du vernaculaire, car ils ne donnent aucun indice au lecteur de la spécificité du terme Jaysis. Ce type d'atténuation constitue essentiellement une approche domestique.

\section{Le poids du passif culturel}

La troisième observation que nous pouvons tirer de ces résultats est que Cohen s'est parfois autorisé l'utilisation de jurons non religieux. Bien que minoritaires, ces jurons reviennent suffisamment pour identifier une inclination différente de sa posture de traducteur.

L'interjection " putain ! ”, en particulier, qu'il emploie à plusieurs reprises vers la fin de l'ouvrage, est un juron français très usité de nos jours, au point que certains se permettent de le prononcer à l'occasion de grands évènements médiatiques : "Ouah, putain, génial, merci ! " s'est exclamé Jean Dujardin, au moment de recevoir l'Oscar du meilleur acteur pour son rôle dans 
The Artist. Comme il s'en est ensuite expliqué, il s'agissait d'un " "putain" lâché comme ça, juste à ce moment-là j'exulte, voilà, je me détends, je me libère(9) ".

Une stratégie consistant à traduire des jurons très usités présents dans le texte source par des jurons très usités de la langue cible peut être considérée comme visant un effet d'équivalence (voir par exemple Nida 1964, Newmark 1981 et Holz-Mänttäri, 1984). II est impossible de vérifier s'il s'agit d'une stratégie délibérée ou non de la part de Cohen mais nous pouvons de nouveau parler d'une forme de domestication (Venuti 1995). Afin de caractériser cette tendance, chaque occurrence a été examinée dans son contexte. La majorité des occurrences se produisent dans le dernier tiers de l'ouvrage. Comme l'exemple ci-dessous l'illustre, "putain ! " est presque toujours employé par le père de Sharon, un homme d'une cinquantaine d'année :

She clouted his arm with the hairbrush.

- Oh Jesus! Me fuckin' - There was no need for tha'.

(Doyle 1990 p. 154)

Et elle planta son peigne dans le bras de son mari.

- Oh putain! Mon bras, putain... Mais qu'est-ce-qui t'a pris ?

(Doyle, traduit par Cohen, 2009 p. 219)

En revanche, "merde ! " est utilisé exclusivement par Sharon et ses amies, en d'autres termes par des femmes de moins de vingt ans :

Her face was really white.

- Oh Jesus, said Jackie. - come on.

(Doyle 1990, p. 206)

Elle avait le visage blanc comme un linge.

- Oh merde, pesta Jackie. Bon viens.

(Doyle, traduit par Cohen, 2009, p. 209).

Le fait que ces jurons non religieux soient utilisés vers la fin du roman pourrait indiquer que cette stratégie n'est pas planifiée mais qu'elle est due, au contraire, à un relâchement de l'autocensure du traducteur. De plus, le choix du juron non religieux semble déterminé par le personnage qui le prononce, une approche que Cohen n'a pas employée dans le cas des jurons religieux. Ce constat est d'autant plus frappant que Doyle ne fait pas de distinction en termes de vulgarité, Jesus, Jaysis et God étant indifféremment utilisés par Sharon ou par son père.

Le traducteur a donc introduit, dans la traduction française, un élément générateur de stéréotype, celui que les femmes n'utilisent pas les mêmes jurons que les hommes. Que cet

(9) Jean Dujardin, Journal de TF1 du 27 février 2012, 20 h 21, disponible sur http://www.telleestmatele.com/ article-video-20-heures-de-tf1-jean-dujardin-revient-sur-le-putain-lache-lors-de-son-discours-100333418.html [visité le 16 février 2013]. 
élément soit fondé ou non, il est spécifique à la traduction française et ne figure pas dans le texte original. Ce fait suppose lui aussi que le traducteur n'a pas fait ce choix consciemment, mais qu'au contraire, il s'est laissé guider par sa perception de l'usage de sa langue maternelle.

En français comme en anglais, l'échelle de grossièreté des jurons reste très subjective. Le dictionnaire Le Robert \& Collins (1995) attribue par exemple deux étoiles à " putain ! " et trois à « merde ! " : deux étoiles marquent " les expressions très familières qui sont à employer avec la plus grande prudence par le lecteur étranger " ( $p$. XXVIII) tandis que trois étoiles marquent "le petit nombre d'expressions courantes que le lecteur étranger doit pouvoir reconnaître, mais dont l'emploi risque d'être ressenti comme fortement indécent ou injurieux " (ibid.). À l'inverse, Le Grand Robert de la langue française (2001), classe " putain! " dans le registre "populaire ", tandis qu'il ne précise pas le registre de " merde ! ". D’autre part, la majorité des personnes consultées dans le cadre de la rédaction du présent article(10) considèrent "putain! " comme plus grossier que " merde! ". Une étude portant sur l'usage de ces jurons par un échantillon d'hommes et de femmes francophones de France, similaire à celle de McEnery (2006), révèlerait peut-être que " putain! " est plus employé par les hommes. Cela reste à prouver et bien qu'une stratégie d'altérité semble avoir été employée pour avertir le lecteur de l'étrangeté du texte original, les seules conclusions que nous pouvons tirer dans le cadre du présent article sont que le traducteur n'a pas totalement échappé à son désir de traduire (Berman 1985/2004), et que sa traduction conforte le lecteur dans sa perception " idéalisée " (cliché) de l'Irlande et dans celle d'un usage langagier sexualisé.

\section{Conclusions}

L'analyse des choix de traduction des termes Jesus, Jaysis et God dans la traduction française du Snapper révèle qu'un certain nombre de clichés ont été introduits.

II semble que le traducteur français ait adopté une stratégie consistant à favoriser l'utilisation de jurons religieux archaïques pour traduire les jurons profanes présents dans le texte source. Cette approche suggère une stratégie d'altérité, alors qu'en réalité, elle consolide un stéréotype bien établi et renforce la domestication.

Dans l'ensemble, cette approche est relativement constante, mais il semble qu'une seconde tendance, qui consiste à employer des termes domestiques très usités, se soit insinuée dans les choix du traducteur. Elle se concentre dans le dernier tiers de la traduction. II est impossible de vérifier si cette tendance correspond à une stratégie délibérée (choix conscient) ou si elle est le simple résultat du désir de traduire (choix inconscient). Néanmoins, dans ce second cas, le choix des termes apparaît directement lié au sexe des personnages qui les prononcent.

(10) La question a été posée à une vingtaine de francophones de France. 
Nous n'avons guère évoqué le plaisir de lire la traduction de Bernard Cohen. Si évident que cela semble, il convient de souligner que le présent article n'est pas une critique littéraire. Bien au contraire, son but est de souligner l'existence d'une stratégie délibérée qui consiste à mettre en relief l'étrangeté du texte traduit. La réussite d'une telle stratégie ne sera jamais totale mais son existence suffit à valider le statut de l'acte de traduction, car le refus du traducteur (du moins ce que nous interprétons comme un refus) d'accepter la stratégie domestique sans condition, peut justifier l'usage du cliché. Bernard Cohen s'appuie avant tout sur le bon sens de son lecteur. En lisant sa traduction, celui-ci se rendra compte que le cliché n'est pas là pour caricaturer les personnages, mais pour faire connaissance avec eux. Le traducteur ne se moque pas de ces personnages mais tente de faire les présentations, et ça fonctionne.

Tableau 1 - Les traductions de Jaysis (44 occurrences) par ordre de récurrence

\begin{tabular}{|c|c|l|}
\hline Nombre & $\%$ & Occurrence \\
\hline 9 & 20 & De Dieu \\
8 & 18 & Doux Jésus \\
7 & 16 & Bon Dieu \\
4 & 9 & Seigneur \\
3 & 7 & Nom de Dieu \\
3 & 7 & Vingt Dieux \\
2 & 5 & Jésus \\
2 & 5 & Mon Dieu \\
1 & 2 & Allons \\
1 & 2 & Bordel \\
\hline
\end{tabular}

Tableau 2 - Les traductions de Jesus (69 occurrences) par ordre de récurrence

\begin{tabular}{|c|c|l|}
\hline Nombre & $\%$ & Occurrence \\
\hline 16 & 23 & Doux Jésus \\
13 & 19 & Seigneur \\
10 & 14 & Bon Dieu \\
9 & 13 & Jésus \\
4 & 6 & Putain \\
3 & 4 & De Dieu \\
2 & 3 & Merde \\
2 & 3 & Mon Dieu \\
2 & 3 & Oh \\
1 & 1 & Allez \\
\hline
\end{tabular}


Tableau 3 - Les traductions de God (39 occurrences) par ordre de récurrence

\begin{tabular}{|c|c|l|}
\hline Nombre & $\%$ & Occurrence \\
\hline 8 & 21 & Mon Dieu \\
6 & 15 & Bon Dieu \\
3 & 8 & Dieu du ciel \\
3 & 8 & Merde \\
2 & 5 & Dieu \\
2 & 5 & Nom de Dieu \\
2 & 5 & Non \\
2 & 5 & Seigneur \\
2 & 5 & Pour l'amour de Dieu \\
1 & 3 & Bon sang \\
\hline
\end{tabular}

\section{Bibliographie}

BERMAN Antoine, 1985. "Translation and the trials of the foreign ". Translated from French by L. Venuti in Lawrence Venuti, ed. 2004, The Translation Studies Reader, Second Edition, London : Routledge, p. 277-289.

BERMAN Antoine, 1986. "La terre nourrice et le bord étranger ". Communications, $n^{\circ} 43$, p. 205-224.

BLANCHOT Maurice, 1971, L'amitié, Paris : Gallimard.

CRYSTAL David, 1995. The Cambridge Encyclopaedia of the English Language. Cambridge: Cambridge University Press.

DELEUZE Gilles et GUATTARI Félix, 1980. "Rhizome ". Capitalisme et schizophrénie. Milles plateaux. Paris : Éditions de Minuit.

DOYLE Roddy, 1990. The Snapper. London: Vintage.

DOYLE Roddy, 1996/2009. The Snapper. Traduit de l'anglais par Bernard Cohen. Paris: Laffont. DUFAYS, Jean-Louis, 2004 « Rumeur et stéréotypie : l'étrange séduction de l'inoriginé ». Protée, vol. 32, $n^{\circ} 3$, p. 25-31.

DUVAL Alain, SINCLAIR KNIGHT Lorna et al., 1995. Le Robert \& Collins Dictionnaire Français< < Anglais senior, Paris : Dictionnaires Le Robert.

FARR Fiona, 2008. Taboo or Not Taboo ? : Swearing and Profane Language Use in Spoken Irish English. Sociolinguistics Symposium, 3-5 avril, université d'Amsterdam.

GUILLERON Gilles, 2007. Le petit livre des gros mots. Paris : Éditions First.

HOLZ-MÄNTTÄRI Justa, 1984. Translatorisches Handeln: Theorie und Methode, Helsinki: Suomalainen Tiedeakatemia. 
IFOP. 2007. Sondage pour La Vie, n 3209, 1er mars 2007.

LITTRÉ Émile, 1873. Dictionnaire de la langue française, Paris : Hachette \& Co.

McENERY Tony, 2006. "How British Men and Women Swear "In Patrick Griffiths, Andrew John Merrison et Aileen Bloomer eds. 2010. Language in Use : a Reader. London : Routledge, pp. 242-255.

NEWMARK Peter, 1981. Approaches to translation, Oxford, Royaume-Uni : Pergamon.

NIDA Eugene 1964. Towards a Science of Translating. Leiden, Pays-Bas : E. J. Brill.

O'ROURKE Bernadette, 2011. "Language ownership in the classroom ". Journal of Language, Identity, and Education, Vol. 10, n 5, novembre-décembre, p. 327-345.

REY Alain, 2001, Le Grand Robert de la langue française, Paris : Dictionnaires Le Robert.

SCHLEIERMACHER Friedrich, 1813. "Ueber die verschiedenen Methoden des Uebersezens". In Hans Joachim Störig, 1963, Das Problem des Übersetzens, Stuttgart, Allemagne : H. Goverts.

SIBLOT Paul, 1995. "Du sens dans les formes exclamatives ", Faits de langues, $n^{\circ}$ 6, septembre, p. 163-170.

STATISTIQUE CANADA, 2001. Population selon la religion, par province et territoire, disponible sur http://www.statcan.gc.ca/tables-tableaux/sum-som/l02/cst01/demo30b-fra.htm [visité le 16 février 2013].

THE GUARD (L'IRLANDAIS), 2011, [film] John Michael McDonagh, Irlande: Reprisal Films, Element Pictures, Crescendo Productions, Aegis Film Fund et Prescience.

VALLÉE Pierre, 2007. "L'Église catholique du Québec - Un patrimoine en danger ", Le Devoir, 7-8 avril 2007, disponible sur http://www.ledevoir.com/societe/ethique-et-religion/138546/ l-eglise-catholique-du-quebec-un-patrimoine-en-danger [visité le 16 février 2013].

VENUTI Lawrence, 1995. The Translator's Invisibility. A history of translation. London: Routledge. VIRILIO Paul, Un paysage d'événements, Paris, Galilée, 1996.

WAJNRYB Ruth, 2005a. Expletive Deleted: A Good Look at Bad Language. New York : Free Press. WAJNRYB Ruth, 2005b. Language most foul. Crows Nest NSW: Allen \& Unwin.

WHITE Caramine, 2001. Reading Roddy Doyle. New York: Syracuse University Press.

Pierre Fuentes, DPLG ARB, MSc (trad.), est architecte et traducteur spécialisé dans les domaines de l'architecture (théorie et pratique), du BTP et de l'immobilier. II a vécu une dizaine d'années en Irlande, avant de s'installer comme traducteur indépendant à Édimbourg en 2009, où il enseigne aussi la traduction vers le français dans le cadre d'un cycle de master à l'université d'Heriot Watt. Site pro: www.pierrefuentes.com 